\title{
Patient characteristics according to rehabilitation and employment status in Korean hemodialysis patients
}

\author{
Jong Yun Lee ${ }^{\mathbb{1}}$, Dong-Chan Jin ${ }^{(\mathbb{D})}$ \\ Department of Internal Medicine, St. Vincent's Hospital, College of Medicine, The Catholic University of Korea, Suwon, Republic of Korea
}

\begin{abstract}
Background: While the final goal of renal replacement therapy is to achieve normal social adaptation and employment, many studies to date have focused on the analysis of complications and causes of death. In contrast, the analysis of dialysis patients with normal job employment and exhibiting a good medical status can be important for clinical treatment policy. This study assessed various clinical parameters according to rehabilitation and employment status to elucidate characteristics correlated with better rehabilitation status among hemodialysis patients.

Methods: A total of 29,865 hemodialysis patients who were registered with rehabilitation status information in the Korean Society of Nephrology end-stage renal disease registration program from January 2017 to April 2019 were included and divided into five groups according to their rehabilitation status.

Results: About half (47\%) of enrolled patients belonged to the "independent but without a job" group and $21 \%$ of patients belonged to the "employed with a full-time job" group. Analysis revealed significantly younger mean age, longer mean hemodialysis duration, a higher proportion of male sex, and a lower proportion of diabetic nephropathy cases in the full-time job group than in the other hemodialysis patient groups. Also, hemoglobin, albumin, and phosphorus levels were higher but the urea reduction ratio was lower in the full-time job group.

Conclusion: A better rehabilitation and employment state of hemodialysis patients was associated with younger age, male sex, and underlying chronic glomerulonephritis. Patients with full-time jobs generally have better laboratory data but lower dialysis efficacy.
\end{abstract}

Keywords: Chronic kidney failure, Employment, Rehabilitation, Renal dialysis

Received March 21, 2020; Revised May 24, 2020;

Accepted June 2, 2020

Editor: Seung-Yeup Han, Keimyung University, Daegu, Republic of Korea

Correspondence: Dong-Chan Jin

Department of Internal Medicine, St. Vincent's Hospital, The Catholic University of Korea, 93 Jungbu-daero, Paldal-gu, Suwon 16247, Republic of Korea. E-mail: jindongc@catholic.ac.kr

Copyright @ 2020 by The Korean Society of Nephrology

(a) This is an open-access article distributed under the terms of the Creative Commons Attribution Non-Commercial License (http://creativecommons. org/licenses/by-nc-nd/4.0/), which permits unrestricted non-commercial use, distribution, and reproduction in any medium, provided the original work is properly cited.

\section{Introduction}

The number of patients with end-stage renal disease (ESRD) has grown rapidly during the last several decades. In 2018, patients on hemodialysis totaled about $80,000-$ or about 1,500 per one million individuals-in the Republic of Korea [1], for a prevalence rate that is one of the highest in the world [2].

Given the rising number of patients requiring dialysis, the quality of dialysis therapy has become of increasingly greater concern. While the final goal of renal replacement therapy is normal social adaptation and employment, most studies of dialysis therapy to date have focused on the analysis of complications and causes of death, with the studies on quality of life, rehabilitation and employ- 
ment status of dialysis patient remaining quite few. In particular, the analysis of dialysis patients with normal job employment and exhibiting a good medical status can be of significance in informing the clinical treatment policy.

Fortunately, a questionnaire about the rehabilitation status of dialysis patients was included in the ERSD registration program of the Korean Society of Nephrology (KSN) and the results are reported annually. This study therefore analyzed various clinical parameters according to rehabilitation and employment status to elucidate the characteristics associated with better rehabilitation status among hemodialysis patients, which could form basic evidence to support the improvement of clinical dialysis therapy in practice.

\section{Methods}

\section{Study population}

The Institutional Review Board of St. Vincent's Hospital approved this study (IRB No. VC19ZISI0222). The number of hemodialysis patients registered in the ERSD registration program of the KSN from January 2017 to April 2019 was 43,856 . Of these, 29,865 hemodialysis patients with information including rehabilitation status and who were 20 years of age or older were enrolled in this study. Study participants were categorized into five groups according to their rehabilitation and employment statuses as follows: group A, full dependence/bed-ridden state (needs help from others with eating, shows difficulty with communicating); group $\mathrm{B}$, partially independent in daily living (can conduct daily life with the help of others, visits the dialysis center with a guardian but toilets alone); group $\mathrm{C}$, independent daily living but no work (unemployed but shows independent daily living, independent meal preparation, visits dialysis center alone); group D, part-time/temporary job (finds it slightly difficult to maintain a job, possibly does housework and family care); and group E, has normal occupational employment (conducts full-time job activities as a normal person except on a dialysis schedule).

\section{Clinical and dialysis parameters}

Baseline demographic data and clinical data including age, sex, height, dry weight, causes of ESRD, duration months of hemodialysis, dialyzer surface area, systolic blood pressure, diastolic blood pressure, various blood chemistry data, normalized protein catabolic rate, urea reduction ratio, residual renal function, and hemodialysis efficacy (single-pool Kt/V) were analyzed as registered data. For patients with repeated registered data, only the last data for each parameter were included in the analysis.

Hemoglobin, serum albumin, urea nitrogen, creatinine, phosphorus, total cholesterol, uric acid, intact parathyroid hormone, and hemoglobin Alc levels were determined from blood samples just before the initiation of a hemodialysis session during the first treatment of the week. All dialysate solutions were bicarbonate-based. The single-pool Kt/V was determined by two-point urea modeling on the basis of an intradialytic reduction in blood urea and ultrafiltration volume during hemodialysis via the Daugirdas equation.

\section{Statistical analyses}

Continuous variables with normal distribution are presented as means \pm standard deviations and those without normal distribution are presented as medians with ranges as appropriate for the type of variable. The one-way analysis of variance, multiple comparison, or Kruskal-Wallis test was used as appropriate to determine differences in continuous variables. Meanwhile, categorical variables are presented as percentages and Pearson's chi-square test was used to determine differences between them. Univariate and multivariate binary logistic regression analyses were also performed for full-time employment (group E vs. other groups). Multivariate binary logistic analysis with backward stepwise analysis (probability for stepwise: entry, 0.05; removal, 0.10) was applied and the results were listed in order of the odds ratio value. All statistical analyses were performed using the SPSS ver. 11.5 software program (SPSS Inc., Chicago, IL, USA).

\section{Results}

\section{Patient characteristics}

Among the total of 29,865 enrolled hemodialysis pa- 
tients, group A (bed-ridden) included 1,717 (5.7\%), group B (dependent daily living) included 5,157 (17.3\%), group C (independent daily living) included 13,983 (46.8\%), group D (part-time job) included 2,687 (9.0\%), and group E (full-time job) included 6,321 (21.2\%) individuals, respectively (Fig. 1). The proportions of male patients in each group were $46.8 \%, 50.3 \%, 58.5 \%, 62.4 \%$, and $76.6 \%$, respectively, which suggests a significantly high proportion of males existed in group E (Fig. 2). The mean ages of each group were $72.9 \pm 11.8,71.8 \pm 11.3,64.0 \pm 12.1$, $57.2 \pm 11.6$, and $54.6 \pm 11.1$ years old, respectively, revealing patients were significantly younger in group $\mathrm{E}$ than in the other groups (Fig. 3, black bar). Conversely, the mean values of hemodialysis duration were 51.9, 54.3,

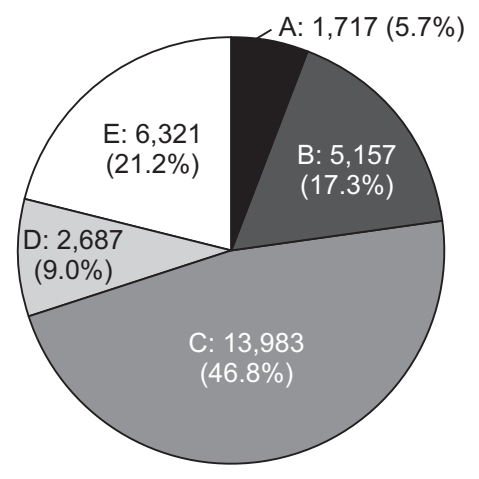

Figure 1. Proportion of the total patient group according to rehabilitation status. Group A, full dependence/bed-ridden state; group $B$, partially independent in daily living; group $C$, independent daily living but no work; group D, part-time job/temporary job; and group E, full-time job and normal work. About half (46.8\%) of enrolled patients belonged to group $\mathrm{C}$ and patients employed in fulltime work (group E) constituted 21.2\%.

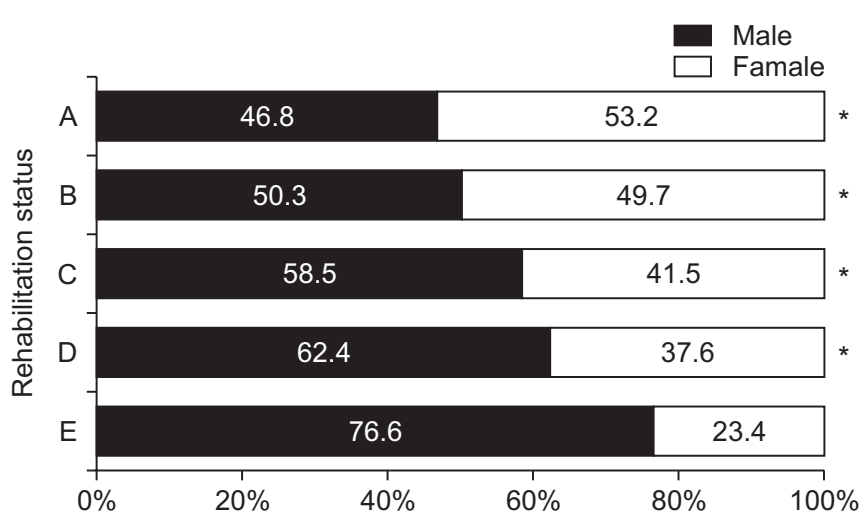

Figure 2. Differences in the rehabilitation status according to sex. Note the big difference in group $E$ (see Fig. 1 for a group definition of rehabilitation status); ${ }^{\star} P<0.01$ versus group $\mathrm{E}$.
64.1, 65.5, and 67.4 months, respectively, highlighting the significantly long duration of dialysis in group E (Fig. 3 , white bar). The underlying cause of ESRD in group $\mathrm{E}$ was diabetic nephropathy in $36.4 \%$, hypertensive nephrosclerosis in $23.4 \%$, and chronic glomerulonephritis in $16.0 \%$ of patients, respectively, showing a significantly low diabetic population when compared with in group A (Fig. 4). The dry body weights were $54.3 \pm 11.3,57.5 \pm$ $10.9,59.7 \pm 11.1,61.9 \pm 12.4$, and $64.0 \pm 12.4 \mathrm{~kg}$, respectively (Fig. 5, bar graph). According to the body weight, values of the inter-dialytic weight gain (i.e., dialyzer surface area) were $1.95 \pm 0.93,2.22 \pm 0.93,2.41 \pm 0.96,2.57 \pm 1.0$,

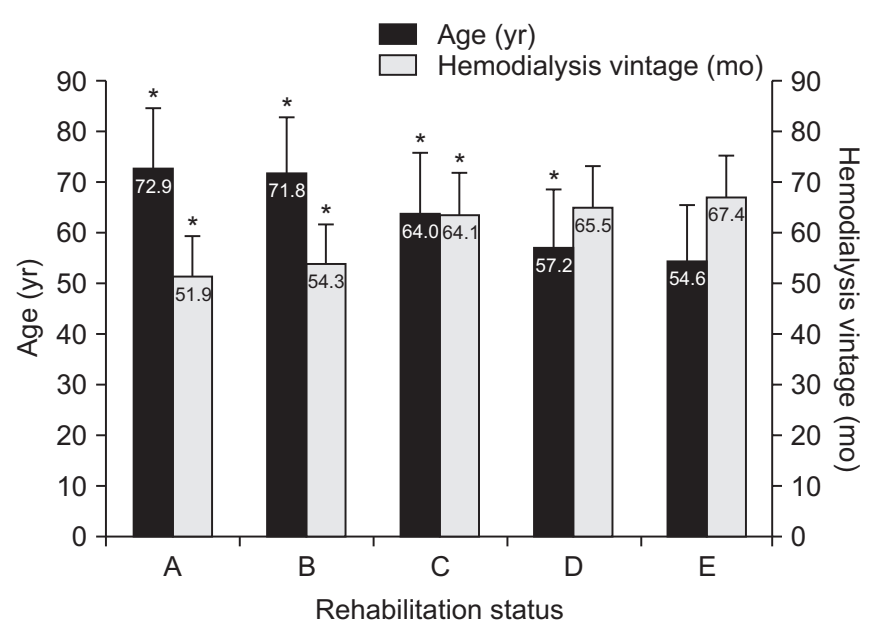

Figure 3. Average age and hemodialysis duration according to the rehabilitation status (see Fig. 1 for a group definition of rehabilitation status); ${ }^{*}<0.05$ versus group $\mathrm{E}$.

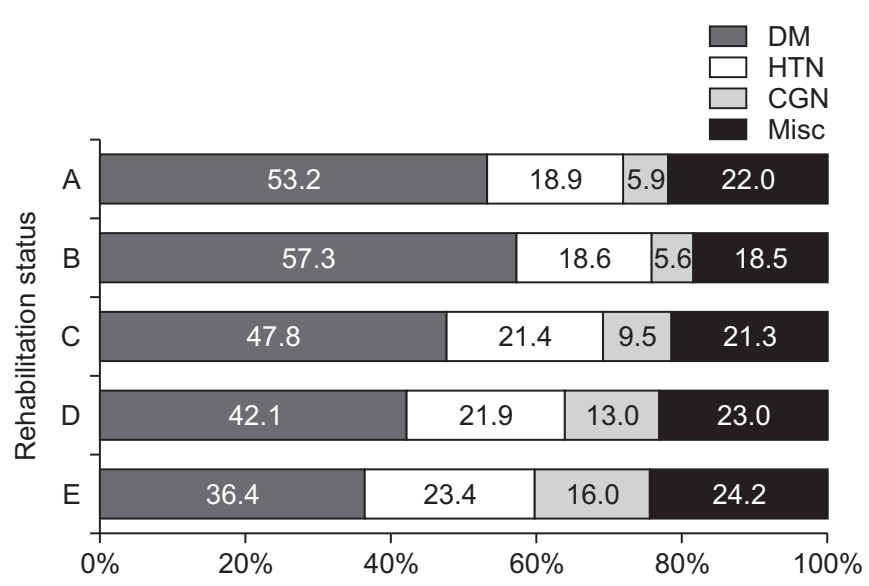

Figure 4. Underlying disease of end-stage renal disease according to the rehabilitation status. Note the small percent of diabetes in group $\mathrm{E}$ (see Fig. 1 for a group definition of rehabilitation status). CGN, chronic glomerulonephritis; DM, diabetic nephropathy; HTN, hypertensive nephrosclerosis; Misc, miscellaneous disease. 
and $2.62 \pm 1.0 \mathrm{~kg}$ (Fig. 5, line graph). The mean values of the dry body weight and dialyzer surface area in group $\mathrm{E}$ were significantly larger relative to in other groups.

\section{Clinical parameters analysis 1: multiple comparisons of means}

The mean systolic blood pressure in group E was $143.7 \pm$ $15.3 \mathrm{mmHg}$, which was significantly higher than that in either group A or B, while the mean diastolic blood pressure in group $\mathrm{E}$ was $80.1 \pm 9.4 \mathrm{mmHg}$, which was also significantly higher than that in group A, B, C, or D (Fig. 6).

Separately, the mean hemoglobin level in group $\mathrm{E}$ was

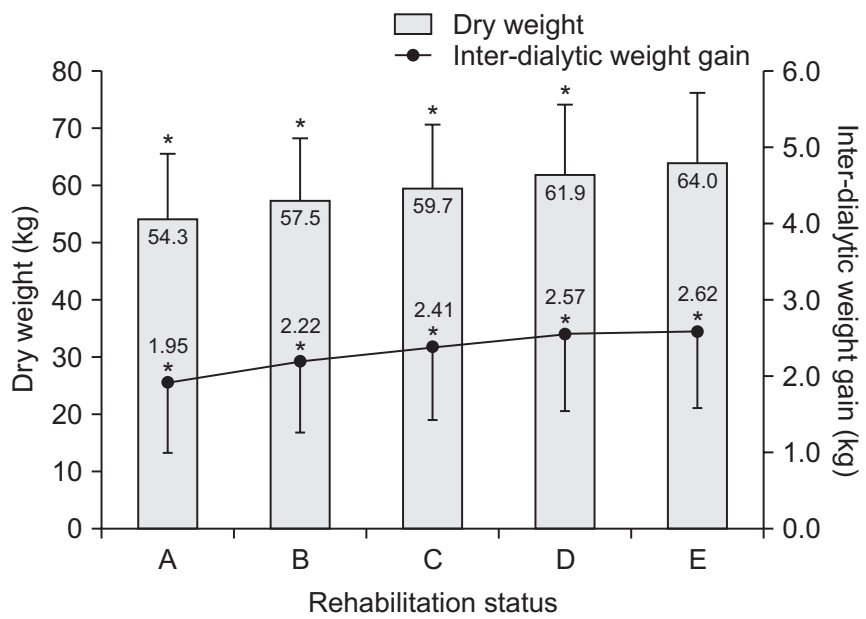

Figure 5. Dry body weight and inter-dialytic weight gain according to the rehabilitation status (see Fig. 1 for a group definition of rehabilitation status); ${ }^{*}<0.01$ versus group $\mathrm{E}$.

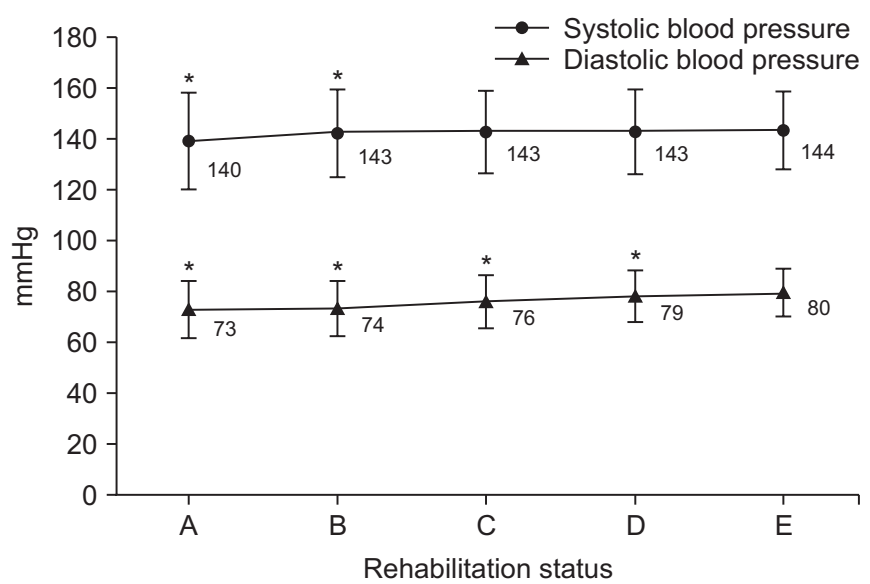

Figure 6. Average blood pressure according to the rehabilitation status (see Fig. 1 for a group definition of rehabilitation status); $P<0.01$ versus group $\mathrm{E}$.
$10.5 \pm 1.0 \mathrm{~g} / \mathrm{dL}$, which was significantly higher than that in group $\mathrm{A}, \mathrm{B}$, or $\mathrm{C}$, while the mean serum albumin level in group $\mathrm{E}$ was $4.1 \pm 0.4 \mathrm{~g} / \mathrm{dL}$, which was significantly higher than that in the other groups. Meanwhile, the mean serum calcium level of group E was $8.8 \pm 0.7 \mathrm{mg} /$ $\mathrm{dL}$, which was not different when compared with in groups $\mathrm{C}$ and $\mathrm{D}$, but the mean serum phosphorus level in group E was $5.4 \pm 1.4 \mathrm{mg} / \mathrm{dL}$, which was significantly higher than that in other groups (Fig. 7). Also, the urea reduction ratio of group $\mathrm{E}$ was $69.9 \% \pm 6.7 \%$, which was significantly lower than as found in other groups and

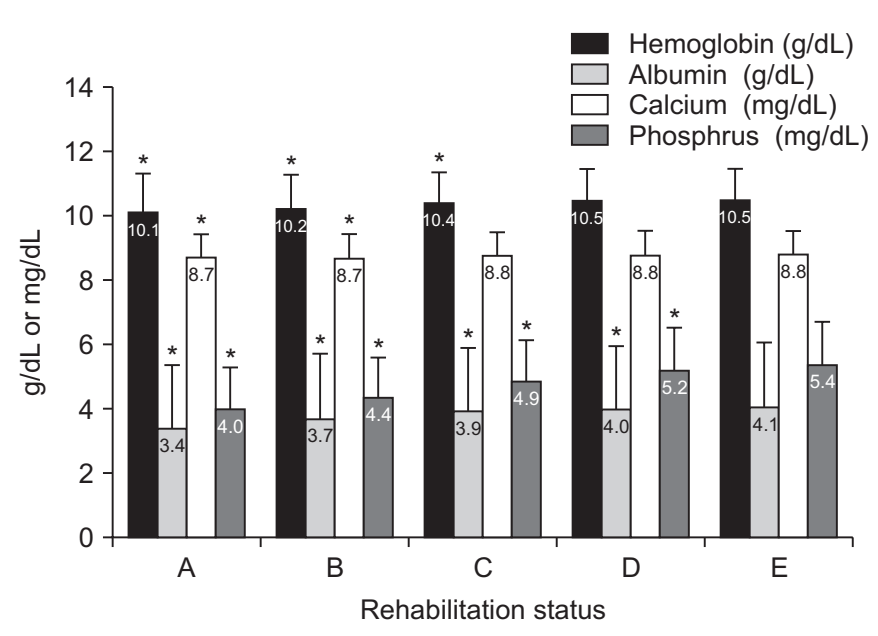

Figure 7. Laboratory data according to the rehabilitation status (see Fig. 1 for a group definition of rehabilitation status); ${ }^{*} P<$ 0.05 versus group $E$.

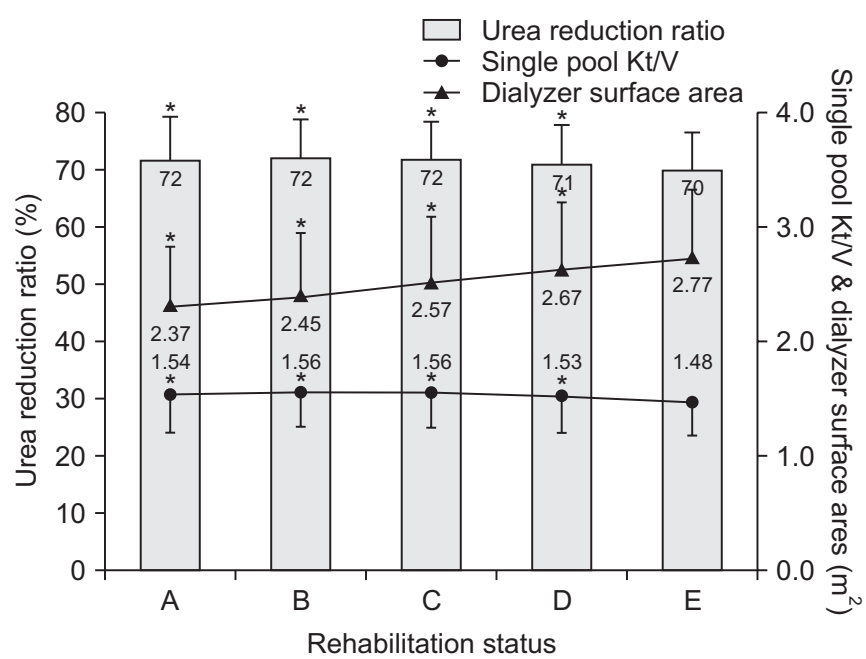

Figure 8. Hemodialysis efficacy and dialyzer surface area according to the rehabilitation status. Note the low hemodialysis efficacy in group $E$ (see Fig. 1 for a group definition of rehabilitation status); $* P<0.01$ versus group $\mathrm{E}$. 


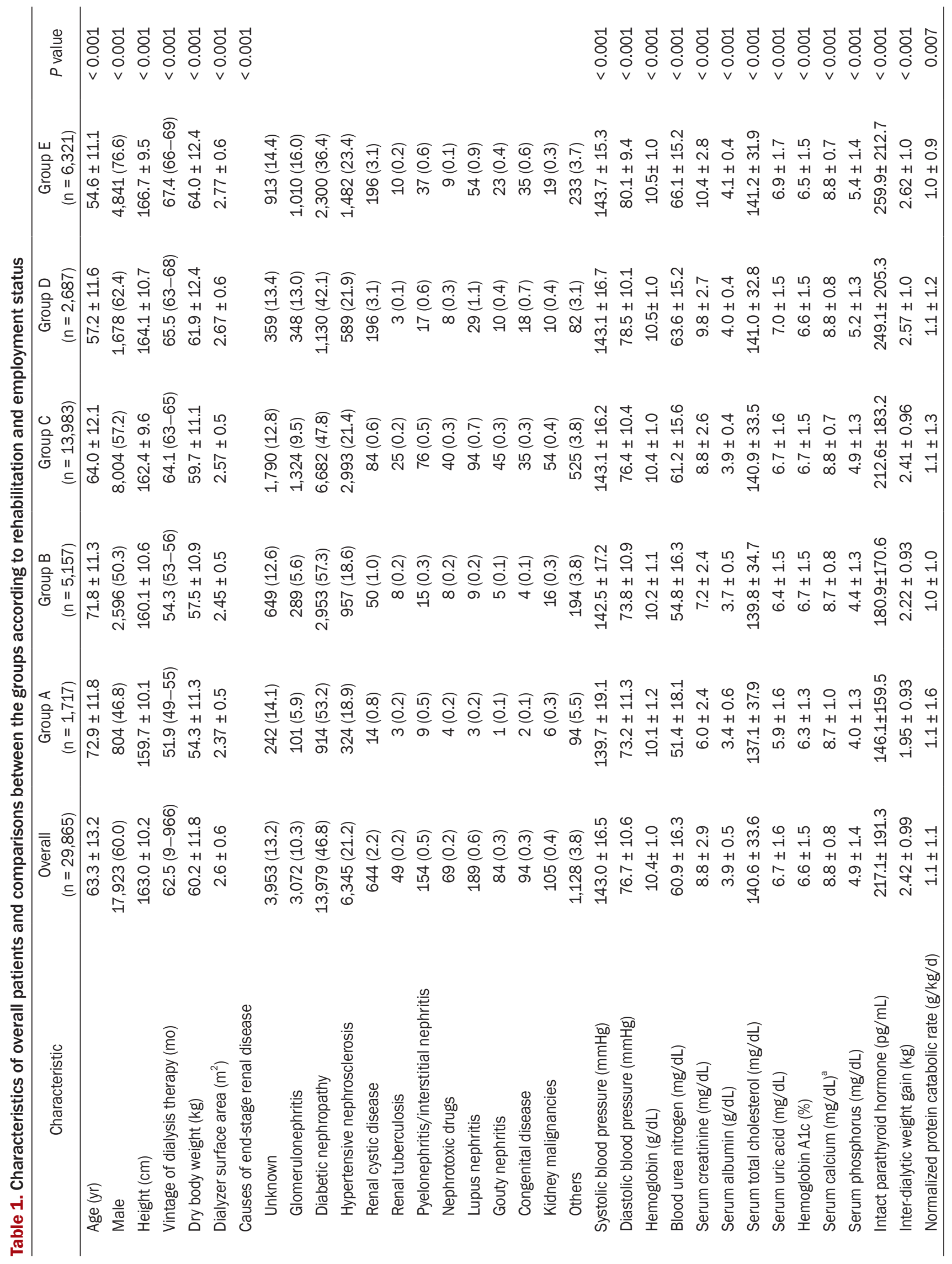




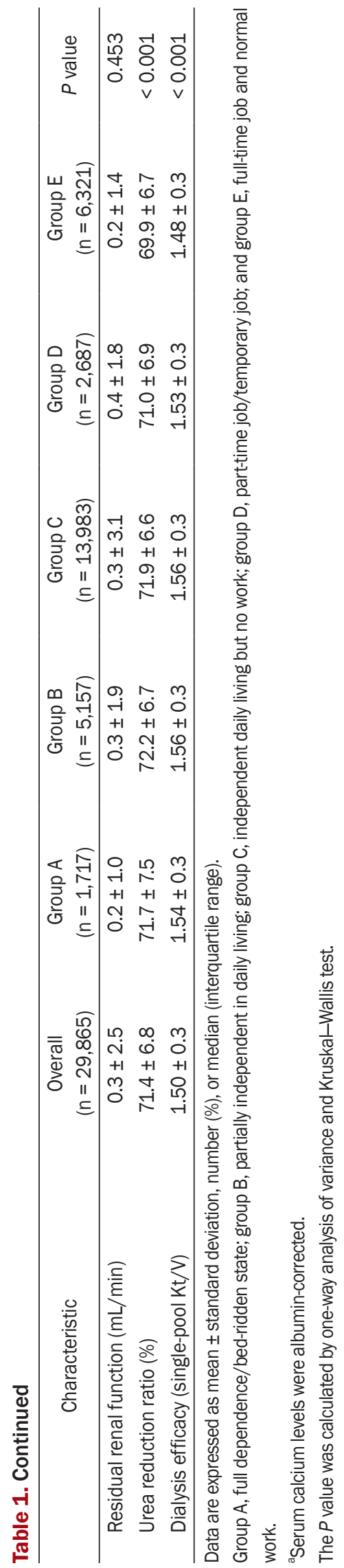

dialysis efficacy (single-pool Kt/V) of group E was $1.48 \pm$ 0.3 , which was significantly lower than values in other groups. Finally, the dialyzer surface area values in each group were $2.37 \pm 0.5,2.45 \pm 0.5,2.57 \pm 0.5,2.67 \pm 0.6$, and $2.77 \pm 0.6 \mathrm{~m}^{2}$ (line graph) (Fig. 8).

\section{Clinical parameters analysis 2: one-way analysis of variance and Kruskal-Wallis test}

There were significant differences between the groups in terms of age, height, hemodialysis vintage, dry body weight, and dialysis surface area by the one-way analysis of variance test $(P<0.001)$. The sex proportion and causes of ESRD among the groups were also significantly different as determined by the Kruskal-Wallis test $(P<$ 0.001) (Table 1).

There were significant differences between all clinical parameters of the groups, such as systolic blood pressure, diastolic blood pressure, hemoglobin, blood nitrogen, serum creatinine, serum albumin, total cholesterol, uric acid, hemoglobin Alc, serum calcium, serum phosphorus, and intact parathyroid hormone results $(P<0.001)$. Considering the dialysis efficacy, normalized protein catabolic rate, urea reduction ratio, and single-pool Kt/V varied significantly among the groups $(P<0.001)$, while residual renal function showed no such difference $(P=$ 0.453) (Table 1).

\section{Clinical parameters analysis 3: logistic regression analyses}

Table 2 lists the results of each clinical parameter's binary logistic regression outcomes for full-time employment (group E vs. other groups). Serum albumin and male sex displayed the highest odds ratios, while dialysis efficacy and diabetic nephropathy were strong negative factors for having full-time employment.

Multivariate (multiple) regression analysis revealed that serum albumin, male sex, and dialyzer surface area had strong positive correlations but dialysis efficacy (spKt/V) and serum calcium had strong negative correlations with full-time employment (Table 3).

\section{Discussion}

The final goal of renal replacement therapy is achieving normal social adaptation and employment, which 
Table 2. Univariate logistic regression analysis for full-time employment

\begin{tabular}{|c|c|c|c|}
\hline & Odds ratio & 95\% confidence interval & $P$ value \\
\hline Age (one-year increments) & 0.935 & $0.933-0.937$ & $<0.001$ \\
\hline Male (versus female) & 2.616 & $2.455-2.788$ & $<0.001$ \\
\hline Vintage of dialysis therapy (by one-month increments) & 1.001 & $1.001-1.002$ & $<0.001$ \\
\hline Dialyzer surface area & 1.994 & $1.898-2.094$ & $<0.001$ \\
\hline Systolic blood pressure (per increment of $10 \mathrm{mmHg}$ ) & 1.003 & $1.002-1.005$ & $<0.001$ \\
\hline Hemoglobin (per increment of $1 \mathrm{~g} / \mathrm{dL}$ ) & 1.145 & $1.114-1.117$ & $<0.001$ \\
\hline Blood urea nitrogen & 1.025 & $1.023-1.027$ & $<0.001$ \\
\hline Serum creatinine & 1.288 & $1.274-1.302$ & $<0.001$ \\
\hline Serum albumin (per increment of $1 \mathrm{~g} / \mathrm{dL}$ ) & 3.576 & $3.318-3.854$ & $<0.001$ \\
\hline Serum cholesterol (per increment of $10 \mathrm{mg} / \mathrm{dL}$ ) & 1.001 & $1.000-1.002$ & 0.101 \\
\hline Intact parathyroid hormone (per increment of $1 \mathrm{pg} / \mathrm{mL}$ ) & 1.001 & $1.001-1.001$ & $<0.001$ \\
\hline Hemoglobin A1c & 0.943 & $0.917-0.969$ & $<0.001$ \\
\hline Inter-dialytic weight gain & 1.344 & $1.301-1.389$ & $<0.001$ \\
\hline Residual renal function & 0.981 & $0.952-1.010$ & 0.189 \\
\hline Normalized protein catabolic rate & 0.951 & $0.908-0.997$ & 0.035 \\
\hline Urea reduction ratio & 0.961 & $0.965-0.966$ & $<0.001$ \\
\hline Dialysis efficacy (spKt/V) & 0.385 & $0.345-0.434$ & $<0.001$ \\
\hline
\end{tabular}

Table 3. Multivariate logistic regression analysis for full-time employment ${ }^{\mathrm{a}}$

\begin{tabular}{|c|c|c|c|}
\hline & Odds ratio & 95\% confidence interval & $P$ value \\
\hline Serum albumin (per increment of $1 \mathrm{~g} / \mathrm{dL}$ ) & 2.764 & $2.149-3.555$ & $<0.001$ \\
\hline Male (versus female) & 2.490 & $2.008-3.086$ & $<0.001$ \\
\hline Serum phosphorus (per increment of $1 \mathrm{mg} / \mathrm{dL}$ ) & 1.086 & $1.006-1.172$ & 0.033 \\
\hline Urea reduction ratio & 1.042 & $1.001-1.085$ & 0.046 \\
\hline Serum cholesterol (per increment of $10 \mathrm{mg} / \mathrm{dL}$ ) & 1.005 & $1.002-1.007$ & 0.001 \\
\hline Vintage of dialysis therapy (by one-month increments) & 0.997 & $0.996-0.999$ & $<0.001$ \\
\hline Systolic blood pressure (per increment of $10 \mathrm{mmHg}$ ) & 0.994 & $0.988-0.999$ & 0.025 \\
\hline Age (one-year increments) & 0.943 & $0.936-0.950$ & $<0.001$ \\
\hline Normalized protein catabolic rate & 0.937 & $0.868-1.011$ & 0.094 \\
\hline
\end{tabular}

${ }^{a}$ In backward stepwise analysis (probability for stepwise: entry, 0.05; removal, 0.10 ), the factors of inter-dialytic weight gain, diabetic nephropathy, uric acid, parathyroid hormone, hemoglobin A1c, diastolic blood pressure, residual renal function, and hemoglobin were removed.

remains a far-off goal for many ESRD patients worldwide [3]. Still, dialysis physicians should keep this goal in mind.
The rapid increase in patients on hemodialysis over the last 30 years is associated with the increase in aged diabetic nephropathy patients, who are rarely enrolled into 
hemodialysis programs [4]. As such, the overall hemodialysis patient's survival and rehabilitation state remain not significantly improved in spite of advances in hemodialysis therapy, such as biocompatible dialyzers and erythropoietin therapy, even increasing the proportion of bed-ridden patients according to a KSN report $[1,4,5]$.

Among enrolled hemodialysis patients in this Korean study, about $21 \%$ of hemodialysis patients had fulltime jobs and $9 \%$ had part-time jobs, while employment rates among hemodialysis patients on dialysis in the United States have been reported to be as low as $18.9 \%$ [6]. A study from Finland reported that the hemodialysis patient employment rate was $19 \%$ on the basis of data from the Finnish Registry for Kidney Diseases, while the employment rate of home dialysis patients was similar to that of transplant recipients and higher than that of in-center hemodialysis patients [7]. There are no home hemodialysis patients in Korea. A recent study from India reported an employment rate of $29.9 \%$ among patients after the initiation of dialysis, with rates of loss of employment of $44 \%$ and $51 \%$ among patients beginning hemodialysis and peritoneal dialysis, respectively [8].

Considering the factors that affect employment rates among patients on dialysis, Muehrer et al [9] reported that maintaining employment at the same level as was seen during the final six months before starting dialysis was more likely to occur among 1) white men aged 30 to 49 years; 2) patients with either glomerulonephritis, cystic, or urologic causes of renal failure; 3 ) patients choosing peritoneal dialysis as their first treatment; 4) those with employer-group or other health plans; and 5) those with erythropoietin usage before ESRD. Patients who underwent treatment for anemia with erythropoiesisstimulating agents before ESRD onset showed higher employment rates at the initiation of dialysis. Meanwhile, a multiple regression analysis showed that age, female sex, and a diagnosis of musculoskeletal disease were independent predictors of poor physical function [10], which were also observed in the present Korean study. Regarding the impact of the dialysis facility, Kutner et al [11] suggested that a higher employment rate was correlated with visitation to a dialysis center available for late evening hemodialysis and home hemodialysis training.

Of note, the KSN data could not confirm the predialysis occupational status, so there was a limitation in evaluating the change by dialysis treatment. Many female di- alysis patients work as homemakers before dialysis and are retained in this manner even after dialysis initiation, which makes evaluation difficult. However, recently, numbers of women with jobs have also increased considerably, especially in the case of unmarried women, while aged men are also retiring early.

To summarize and conclude this study, as expected, young male nondiabetic patients had higher employment rates and patients with full-time job had higher blood pressure, higher hemoglobin and serum factors, and lower dialysis efficiency. These aspects are difficult to control clinically and it is likely that it is important to increase the dialysis efficacy and to control serum phosphorus in young male patients who are working.

\section{Conflicts of interest}

All authors have no conflicts of interest to declare.

\section{Acknowledgments}

The authors wish to thank the KSN ESRD registry committee for the data used in this study.

\section{Authors' contributions}

Jong Yun Lee participated in the statistical analysis and wrote the manuscript. Dong-Chan Jin designed the study and performed the data analysis, wrote the manuscript. Both authors read and approved the final manuscript.

\section{References}

[1] ESRD Registry Committee, Korean Society of Nephrology. Current renal replacement therapy in Korea 2018 [cited 2020 Mar 15]. Available from: http://www.ksn.or.kr/eng.

[2] 2018 USRDS annual data report [Internet]. Bethesda (United States): United States Renal Data System, 2018 [cited 2020 Mar 15]. Available from: http://www.usrds.org/.

[3] Hallab A, Wish JB. Employment among patients on dialysis: an unfulfilled promise. Clin J Am Soc Nephrol 2018;13:203204.

[4] Jin DC, Yun SR, Lee SW, et al. Current characteristics of dialysis therapy in Korea: 2016 registry data focusing on diabetic patients. Kidney Res Clin Pract 2018;37:20-29.

[5] Jin DC. Analysis of mortality risk from Korean hemodialysis 
registry data 2017. Kidney Res Clin Pract 2019;38:169-175.

[6] Erickson KF, Zhao B, Ho V, Winkelmayer WC. Employment among patients starting dialysis in the United States. Clin J Am Soc Nephrol 2018;13:265-273.

[7] Helanterä I, Haapio M, Koskinen P, Grönhagen-Riska C, Finne P. Employment of patients receiving maintenance dialysis and after kidney transplant: a cross-sectional study from Finland. Am J Kidney Dis 2012;59:700-706.

[8] Lakshmi BS, Kumar ACV, Reddy HK, et al. Employment status of patients receiving maintenance dialysis- peritoneal and hemodialysis: a cross-sectional study. Indian J
Nephrol 2017;27:384-388.

[9] Muehrer RJ, Schatell D, Witten B, Gangnon R, Becker BN, Hofmann RM. Factors affecting employment at initiation of dialysis. Clin J Am Soc Nephrol 2011;6:489-496.

[10] Blake C, Codd MB, Cassidy A, O'Meara YM. Physical function, employment and quality of life in end-stage renal disease. J Nephrol 2000;13:142-149.

[11] Kutner N, Bowles T, Zhang R, Huang Y, Pastan S. Dialysis facility characteristics and variation in employment rates: a national study. Clin J Am Soc Nephrol 2008;3:111-116. 